\title{
An SMS fiber structure based on chalcogenide multimode fiber
}

\author{
Pengfei Wang ${ }^{1,2^{*}}$, Gilberto Brambilla ${ }^{1}$, Ming Ding ${ }^{1}$, Xueliang Zhang ${ }^{1,3}$, Yuliya Semenova ${ }^{2}$, Qiang \\ $\mathrm{Wu}^{2}$, and Gerald Farrell ${ }^{2}$ \\ ${ }^{1}$ Optoelectronics Research Centre, University of Southampton, Southampton SO17 1BJ, United \\ Kingdom \\ ${ }^{2}$ Photonics Research Centre, Dublin Institute of Technology, Kevin Street, Dublin 8, Ireland \\ ${ }^{3}$ College of Optoelectronic Science and Engineering, National University of Defense Technology, \\ Changsha 410073, China
}

\begin{abstract}
We theoretically and experimentally investigate a singlemode-multimode-singlemode (SMS) structure based on chalcogenide $\left(\mathrm{As}_{2} \mathrm{~S}_{3}\right)$ multimode fiber and conventional silica singlemode fibers. The experimental results show a general agreement with the numerical simulation results based on a wide angle-beam propagation method (WA-BPM). The chalcogenide fiber and silica fibers were mechanically spliced and packaged using a UV cured polymer with a low refractive index on a microscope slide. Multimode interference variation was observed by photo-induced refractive index changes resulting from both a localized laser irradiation at a wavelength of $405 \mathrm{~nm}$ and a UV lamp. Our result provides a platform for the development of compact, high-optical-quality, and robust photonic nonlinear devices.
\end{abstract}

Keywords: Fiber optics, Multimode interference, Nonlinear fiber, Photosensitivity

\section{INTRODUCTION}

Multimode interference (MMI) has been intensively investigated in photonic integrated waveguides and the unique performance of self-imaging of the input light field is well known and widely employed in beam splitters, combiners and multiplexers for optical communications [1-3]. Recently, multimode interference occurring in a single-mode-multimode-single-mode (SMS) fiber structure has been studied for applications in novel optical devices, e.g., displacement sensor [4], stain and temperature sensor [5], refractometer sensor [6] and edge filter for wavelength measurements [7]. These optical devices, based on such an SMS fiber structure, offer all-fiber solutions for optical communications and optical sensing with the advantages of ease of packaging and connection to optical fiber system.

Chalcogenides are rapidly establishing themselves as technologically superior materials for emerging applications in non-volatile memory and high speed switching [8] and have been considered for a range of other optoelectronic technologies. Chalcogenide glasses offer a wealth of attractive properties such as exceptionally high nonlinearity, photosensitivity, ultrafast nonlinear response, a low phonon energy matrix, the ability to be doped with active elements including lanthanides and transitional metals and the possibility to form detectors, lasers and amplifiers. Unlike any other optical material, they have been formed into a multitude of shapes, including optical fibers, thin films, bulk optical components, microsphere resonators, metamaterials and nanoparticles, patterned by CMOS compatible processing at the sub-micron scale. To date, applications including ultrafast all-optical switching, supercontinuum generation, broadband

*pw3y09@orc.soton.ac.uk; phone: +44 23 8059 3954; fax: +44 2380593149 
wavelength conversion, all-optical signal processing, ultrafast pulse characterization and Raman fiber lasers have been demonstrated extensively using chalcogenide glass fibers [9-11].

In this paper, we present an investigation of light propagation within an SMS fiber structure based on chalcogenide and silica fibers, which has not been addressed so far in the literature. The chalcogenide fiber based multimode interference device is fabricated and packaged by using UV curing. Due to the photo-induced refractive index changes in the chalcogenide glass material, the spectral response achieved for multimode interference also varies with both power and irradiation position for a localized laser irradiation with a maximum output power of $10 \mathrm{~mW}$ at a wavelength of $405 \mathrm{~nm}$. The peak shift of the spectral responses of $2 \mathrm{~nm}$ has been realized and we also achieved a power variation at $1565.4 \mathrm{~nm}$ as high as $8.94 \mathrm{~dB}$ depending on different localized laser irradiation positions along the chalcogenide fiber. The fabricated device offers the potential for low-cost, robustly assembled fully integrated all-optical switching and tunable filter devices due to its unique high nonlinearity and ease of fabrication.

\section{THEORETICAL ANALYSIS}

An SMS fiber structure consists of input and output silica singlemode fibers with a short section of multimode fiber sandwiched between them as shown in Fig. 1. A chalcogenide multimode fiber section was sandwiched between two standard silica singlemode fibers and forms a typical fiber multimode interferometer.

Chalcogenide multimode

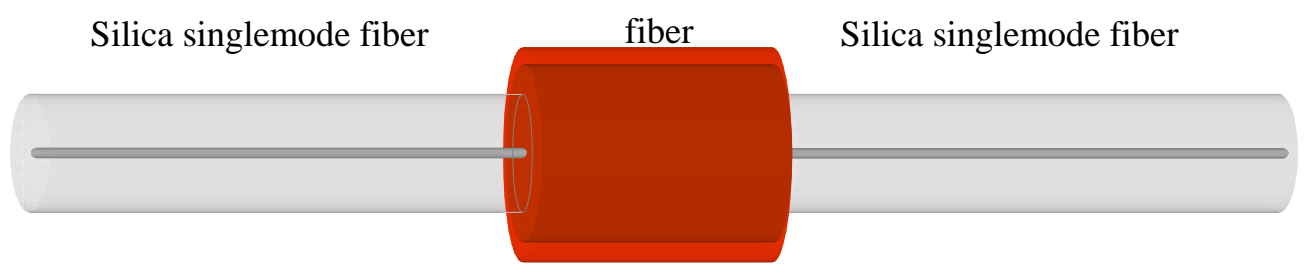

Figure 1. Schematic of an SMS fiber structure based on silica singlemode fiber-chalcogenide multimode fiber-silica singlemode fiber.

Based on the numerical models using a WA-BPM presented in [12], Fig. 2 (a) and (b) present the amplitude distributions of the propagating optical fields and the corresponding coupling loss to the output singlemode fiber as a function of propagation distance for the MMI device. From Fig. 2 (a) and (b), one can see that the first significant multimode interference for the numerical sample of MMF occurs when the propagation distance is longer than $10 \mathrm{~mm}$ and the coupling loss reaches a maximum value of $-50 \mathrm{~dB}$ at a propagation position of $\mathrm{Z}=18.91 \mathrm{~mm}$. The calculated results show that the eigenmode interference within the MMF section is determined by both the size and the refractive index of the MMF core. 

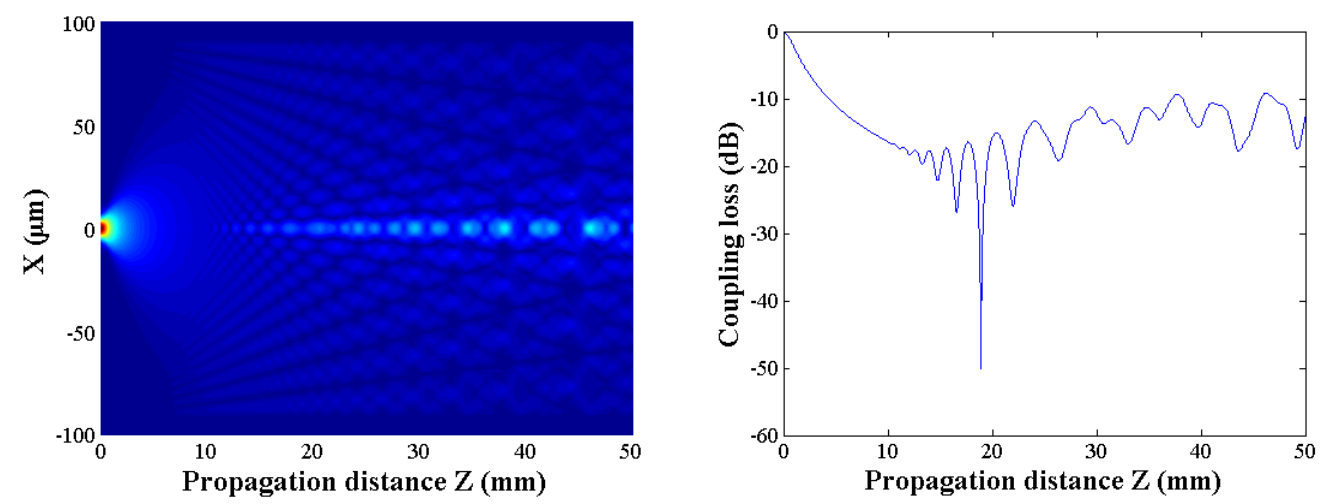

Fig. 2. (a) Light propagation within the chalcogenide multimode fiber; (b) Calculated coupling loss to output singlemode fiber for different chalcogenide multimode fiber lengths when the wavelength of input light is $1550 \mathrm{~nm}$.

\section{EXPERIMENTAL RESULTS AND DISCUSSION}

The chalcogenide fiber used in the experiments is a commercial step-index multimode fiber provided by Oxford Electronics, with an $\mathrm{As}_{2} \mathrm{~S}_{3}$ core $(\mathrm{OD}=180 \mu \mathrm{m})$ and $\mathrm{As}_{\mathrm{x}} \mathrm{S}_{1-\mathrm{x}}$ cladding of lower refractive index $(\mathrm{OD}=275 \mu \mathrm{m})$. The chalcogenide multimode fiber cross section is shown in Fig 3.

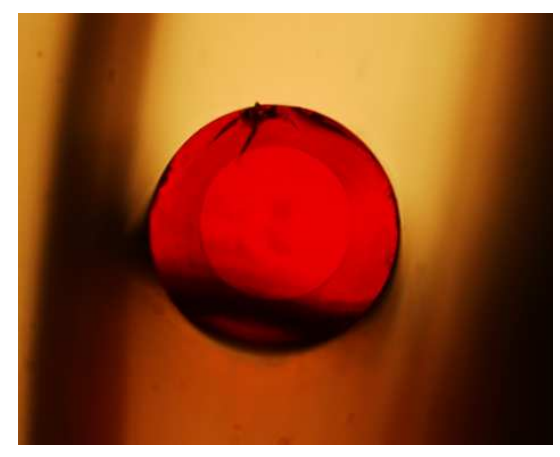

Fig. 3. Microscope image of chalcogenide multimode fiber cross section.

The SMS sample was manufactured by sandwiching the commercial chalcogenide multimode fiber between two silica single mode fibers (SMF). The fiber cores were aligned using 3D translation stages, and alignment was fixed by embedding the fibers in UV curable polymer with a low refractive index. Figure 4 shows the resulting hybrid SMS structure manufactured from a $48.4 \mathrm{~mm}$ long chalcogenide multimode fiber packaged on a microscope slide. The transmission spectra of the fabricated hybrid SMS device were then recorded using a broadband Erbium Doped Fiber Amplifier (EDFA) source (1520 1570 nm) and an optical spectrum analyzer (YOKOGAWA AQ6370). 


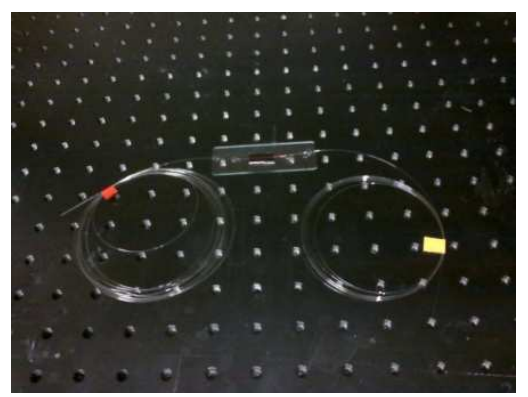

Fig. 4. Image of a chalcogenide multimode fiber with a length of circa $48.4 \mathrm{~mm}$ sandwiched between two silica singlemode fibers and packaged on a microscope slide.

Figure 5 (a) presents the calculated and measured transmission spectra of the hybrid SMS structure: the measured insertion loss is circa $-13 \mathrm{~dB}$. Results show a general agreement with theoretical predictions. The discrepancy between the calculated and measured results could be due to both the alignment between SMFs and MMF and the approximations made in the calculation (simulated transmission for an SMS includes some approximations, such as the Padé $(3,3)$ approximate operator). In order to demonstrate the stability of the SMS fabricated, Fig. 5 (b) presents the transmission spectra of SMS device as they vary with time before/after the UV glue curing process. The changes in the transmission spectra are due to the temperature change and further curing of the polymer glue.

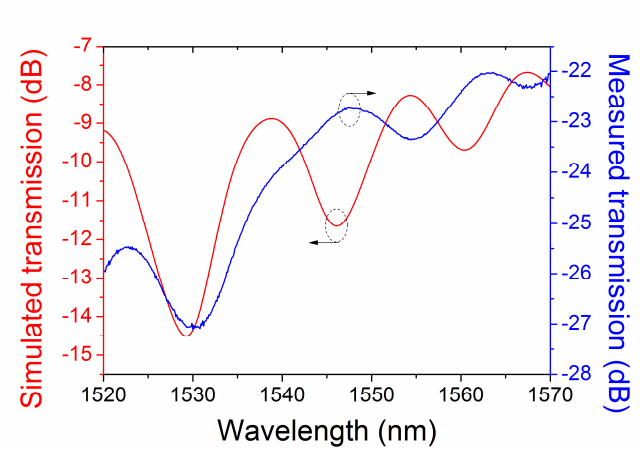

(a)

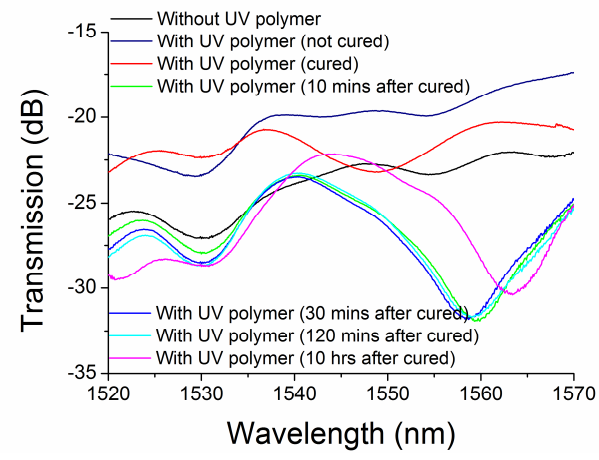

(b)

Figure 5. (a) Calculated and measured spectral responses of the hybrid SMS device over a wavelength range of 1520 1570 nm; (b) change in the SMS transmission spectra before/after UV glue curing.

It is well known that a chalcogenide glass has a high photosensitivity, and thus its refractive index can vary with external laser irradiation. Therefore the spectral response achieved from the multimode interference within the SMS device can also vary with a laser irradiation. To demonstrate this effect, the chalcogenide MMF were irradiated and scanned by a localized CW laser with a maximum output power of $10 \mathrm{~mW}$ and operating wavelength of $405 \mathrm{~nm}$. The laser spot size on the chalcogenide MMF was estimated to be circa $7 \times 10^{-4} \mathrm{~cm}^{2}$, providing an irradiation intensity of $1.43 \times 10^{4} \mathrm{~mW} / \mathrm{cm}^{2}$. The resulting changes in the transmission spectrum were monitored by the OSA in real time. Fig. 6 shows that the spectral 
response red-shifts by $2 \mathrm{~nm}$ and power variations as high as $8.94 \mathrm{~dB}$ at $\lambda=1565.4 \mathrm{~nm}$ were observed for different localized laser irradiation positions along the chalcogenide fiber. The entire chalcogenide MMF was then irradiated by a UV lamp (Hamamatsu L9588-02A, wavelength range: $240-400 \mathrm{~nm}$ ) with an average output power of $410 \mathrm{~mW} / \mathrm{cm}^{2}$. Fig. $6 \mathrm{shows}$ that significant changes of the SMS spectrum occur and a power variation at $1563.3 \mathrm{~nm}$ as high as $7.57 \mathrm{~dB}$ can be achieved.

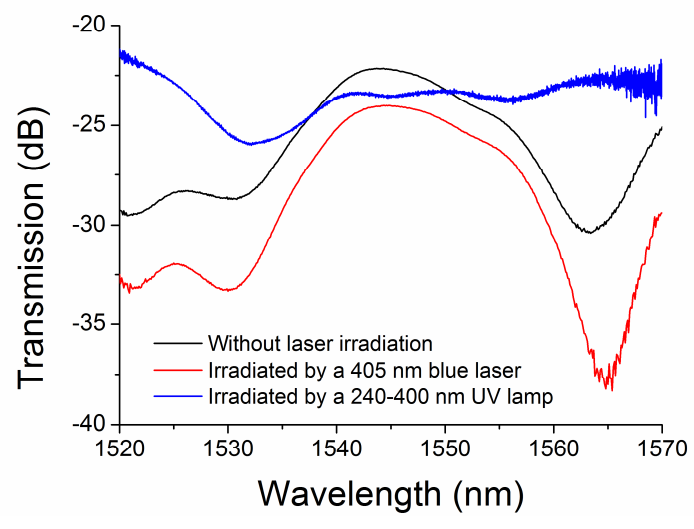

Figure 6. Transmission spectra of the chalcogenide SMS device before/after irradiation by a $405 \mathrm{~nm}$ laser and a UV lamp.

\section{CONCLUSION}

In conclusion, as a first proof of concept, we have proposed and demonstrated a chalcogenide MMF based SMS structure. An acceptable agreement between the experimental characterization and numerical simulation of the structure has been realized. Due to the high photosensitivity of the chalcogenide glass material itself, the chalcogenide SMS structure can be utilized to form a range of functionalities over near- and mid-IR wavelength ranges, such as tunable filter and all-optical switching fiber device. This geometry may also be considered as a promising platform for linear signal processing devices with thresholds orders of magnitudes lower compared to conventional silica based fiber devices.

\section{ACKNOWLEDGEMENT}

P. Wang is funded by the Irish Research Council for Science, Engineering and Technology, co-funded by the Marie-Curie Actions under FP7, G. Brambilla gratefully acknowledges the Royal Society (London) for his research fellowship, Q. Wu gratefully acknowledges the support of Science Foundation Ireland under grant no. 07 /SK/I1200.

\section{REFERENCES}

[1]. Soldano, L. B. and Pennings, E. C. M., "Optical Multi-Mode Interference Devices Based on Self-Imaging: Principles and Applications", J. Lightwave Tech., 13(4), 615-627 (1995). 
[2]. Herben, C. G. P., Vreeburg, C. G. M., Leijtens, X. J. M., Blok, H., Groen, F. H., Moerman, I., Pedersen, W. and Smit, M. K., "Chirping of an MMI-PHASAR Demultiplexer for Application in Multiwavelength Lasers", IEEE Photonics Tech. Lett., 9(8), 1116-1118 (1997).

[3]. Paiam, M. R. and MacDonald, R. I., "A 12-channel phased-array wavelength multiplexer with multimode interference couplers", IEEE Photonics Tech. Lett., 10(2), 241-243 (1998).

[4]. Wu, Q., Semenova, Y., Wang, P., Hatta A. M. and Farrell, G., "Experimental demonstration of a simple displacement sensor based on a bent single-mode-multimode-single-mode fiber structure", Meas. Sci. Technol., 22, 025203 (2011).

[5]. Tripathi, S. M., Kumar, A., Varshney, R. K., Kumar, Y. B. P., Marin, E. and Meunier, J.-P., "Strain and Temperature Sensing Characteristics of Single-Mode-Multimode-Single-Mode Structures," Journal of Lightwave Technology , 27(13), 2348-2356 (2009).

[6]. Wang, P., Brambilla, G., Ding, M., Semenova, Y., Wu, Q. and Farrell, G., “A high sensitivity, evanescent field refractometric sensor based on tapered multimode fiber interference”, Optics Letters, 36(12), 2233-2235 (2011).

[7]. Wang, Q., and Farrell G., "Multimode Fiber based Edge Filter for Optical Wavelength Measurement Application", Microwave and Optical Technology Letters, 48(5), 900-902 (2006).

[8]. Grillet, C., Bian, S. N., Magi, E. C. and Eggleton, B. J., "Fiber taper coupling to chalcogenide microsphere modes," Appl. Phys. Lett., 92, 171109 (2008).

[9]. Asobe, M., Kobayashi, H., Itoh, H. and Kanamori, T. "Laser-diode-driven ultrafast all-optical switching by using highly nonlinear chalcogenide glass fiber," Opt. Lett., 18(13), 1056-1058 (1993).

[10]. Shakeri, S., and Hatami, M., "Self-tunable chalcogenide Raman laser," J. Opt. Soc. Am. B, Vol. 27(4), 679-684 (2010).

[11]. Eggleton, B. J., Luther-Davies, B. and Richardson, K., "Chalcogenide photonics”, Nature Photonics, 5, 141-148 (2011).

[12]. Wang, P., Brambilla, G., Ding, M., Semenova, Y., Wu, Q. and Farrell, G., "Investigation of singlemode-multimode-singlemode and singlemode-tapered multimode-singlemode fiber structures and their application for refractive index sensing," Journal of the Optical Society of America B, 28(5), 1180-1186 (2011). 